\section{Highlights of the NCCN Oncology Research Program}

The NCCN Oncology Research Program (ORP) strives to improve the quality of life for patients and reduce cancer-related deaths by advancing cancer therapies through research. Since the program's establishment in 1999, the NCCN ORP has brought millions of dollars in research grants to investigators at NCCN Member Institutions. Research grants are provided to NCCN through collaborations with pharmaceutical and biotechnology companies; these grants are in turn used to support scientifically meritorious cancer research efforts.

NCCN ORP studies typically explore new avenues of clinical investigation and seek answers to important cancer-related questions. All studies are approved and funded through a scientific peer-review process and are overseen by the ORP.

An NCCN study funded through the grant mechanism is highlighted below.

\section{Phase II Study of Ofatumumab as Front-line Treatment in Elderly, Unfit Patients With Chronic Lymphocytic Leukemia}

\section{Principal Investigator: Alessandra Ferrajoli, MD, BS}

Condition: Chronic lymphocytic leukemia

Institution: The University of Texas MD Anderson Cancer Center

Ofatumumab, a novel anti-CD20 antibody, has been tested in various hematologic malignancies, and is currently FDA-approved for the treatment of patients with chronic lymphocytic leukemia (CLL) that is relapsed/refractory to prior treatments with fludarabine and alemtuzumab.

The goal of thi s clinical research study is to learn whether ofatumumab can help to control CLL. The safety of this drug will also be studied.

\section{Primary Objective:}

- Evaluate the overall response rate of ofatumumab in the upfront setting in elderly, unfit patients with CLL

\section{Secondary Objectives:}

- Determine the overall survival with ofatumumab in the upfront setting in elderly, unfit patients with CLL

- Evaluate the complete response rate and time to progression after repeated doses of ofatumumab in elderly, unfit patients with CLL

- Determine the plasma levels of ofatumumab in elderly, unfit patients

- Evaluate predictive capability of miRNAs detection in plasma samples

Contact: Alessandra Ferrajoli, MD, BS • 713-792-2063 • aferrajo@mdanderson.org

ClinicalTrials.gov Identifier: NCT01444716
The goal of the Highlights of the NCCN Oncology Research Program (ORP) is to provide readers with more information on the ORP, including studies currently accruing patients.

For more information on specific trials, including patient selection criteria, please use the contact information listed with each study.

For more information on the NCCN ORP, including a complete detailing of the clinical studies currently underway at NCCN Member Institutions, please access the NCCN ORP pages at NCCN.org/clinical_trials/ clinicians.asp. 